\title{
Hybrid Protocol for Reducing Interference in VANETS
}

\author{
Khyati Jain \\ K.I.E.T, \\ Ghaziabad \\ U.P, India
}

\author{
Utkarsh Singh \\ K.I.E.T, \\ Ghaziabad \\ U.P, India
}

\author{
Preeti Singh \\ K.I.E.T, \\ Ghaziabad \\ U.P, India
}

\author{
Arvind Kumar \\ K.I.E.T, \\ Ghaziabad \\ U.P,India
}

\author{
Arun Kumar \\ Tripathi \\ K.I.E.T, \\ Ghaziabad \\ U.P, India
}

\begin{abstract}
The Vehicular Ad-Hoc Network (VANET) is a subset of Mobile Ad-Hoc Network (MANET). In this technology moving cars are used as nodes to create a mobile network. Every participating car in VANET is turned into a wireless router or node and is allowed to connect and create a network with other such nodes and the ROAD SIDE UNITS (RSUs) called the Access Points (APs). Message passing through wireless network is the only possible way of communication among these moving nodes. Various clustering algorithms have been suggested by researchers for message passing. In a VANET scenario the timely delivery of useful messages is of as much importance as receiving those messages is. This can be achieved by decreasing the number of messages exchanged in the network. For this a hybrid scheme is suggested, in which the Access Points (AP) are made the Cluster Heads (CHs) which are further connected to routers that store the information regarding the nodes. Making the APs as $\mathrm{CHs}$ eliminates the $\mathrm{CH}$ selection process which decreases the number of messages passed and hence reducing the interference and delay in message delivery caused by the flooding of unnecessary messages received to a node.
\end{abstract}

\section{General Terms}

Interference Reduction, VANET, Intelligent Transportation System (ITS), Networking, Routing Protocols, Clustering Algorithm.

\section{Keywords}

Vehicular Ad-Hoc Network (VANET), Interference Reduction, Intelligent Transportation System (ITS), Cluster Head $(\mathrm{CH})$, and Access points (AP), nodes, cars, message passing routers.

\section{INTRODUCTION}

Various researches have been done in communication and computer technology to improve mode of transportation. The purpose of these transportation technological advancements, called Intelligent Transportation System (ITS), is to improve the safety, efficiency, dependability and cost effectiveness of our transportation system thus reducing the number of miss happenings. Advancements in wireless communication that made communication between RSU (access points) and moving nodes (cars) possible, gave birth to a new technology namely Vehicular Ad-Hoc Network(VANET). There are basically two types of communication processes taking place in the system.

\subsection{Vehicle to vehicle communication (V2V)}

Two or more moving nodes communicate amongst themselves with message passing within or outside a cluster.

\subsection{Vehicle to Infrastructure communication (V2I)}

A node communicates with the RSUs and shares all the necessary information with them.

For proper message passing or routing various clustering algorithms are being used. The basic idea about clustering algorithm is that it works by splitting the long stream of vehicles on the Highway Model into small "groups or chains of vehicles" while keeping in mind our goal of interference reduction. These chains are our clusters. A large number of messages are flooded to any node while it is in movement out of which many messages are not of its use but cause delay in the delivery of useful messages. This is called interference and needs to be reduced for the timely delivery of useful messages. Each node depending upon various factors chooses its cluster and thus exchanges messages accordingly. Each cluster consists of a $\mathrm{CH}$ which is expected to be most stable and having the fastest Internet connection in the cluster. In case of communication within the cluster the cluster nodes may either communicate directly with each other or in case of wider distance amongst the two send it via $\mathrm{CH}$. On the other hand when a node needs to send a message to the node of another cluster it needs to send the message to its $\mathrm{CH}$ which further passes it on to the $\mathrm{CH}$ of the specified node's cluster. Figure 1 shows the on road network of VANET.

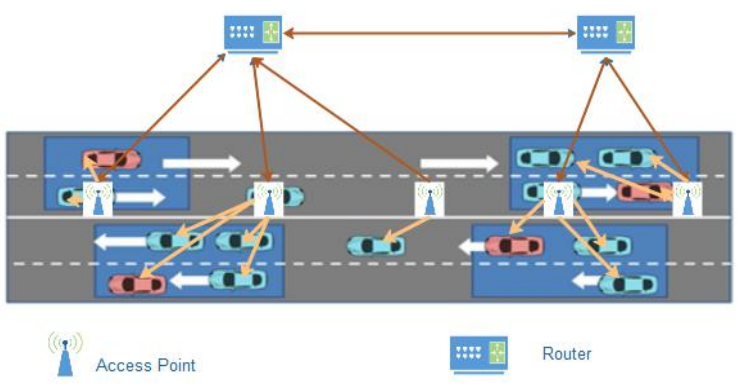

Fig 1: On road VANET network

VANET applications such as emergency warning system, lane-changing assistant, traffic sign/signal violation warning and intersection coordination usually demand direct communication among nodes due to the stringent delay requirements. The main problem that arises here is flooding i.e. Because of the shared wireless medium, blindly and carelessly broadcasting of the packets lead to large number of packet broadcasts which may lead to frequent transmission collisions among neighboring nodes. Thus sending such messages from end to end must be as fast as possible which is possible only by reducing the number of messages by reducing the network interference.

Therefore, in this paper an idea for enhancing the present messaging technique by clustering algorithm is proposed. 
It is suggested that by making an access point as the permanent $\mathrm{CH}$ we can reduce the number of messages shared in analyzing and identifying a new $\mathrm{CH}$ after regular time intervals. The cluster nodes may identify their new $\mathrm{CHs}$ as soon as they enter the range of the next cluster. Thus this improves the stability of the network and lowers the messaging cost, waiting time and packet loss of the useful information.

The remainder of this paper is organized as follows. Section II presents the literature survey on broadcasting of messages in VANET. Section III describes the Hybrid network that is proposed in this paper. Section IV discusses the comparative advantages of our proposal over the earlier used techniques. Finally, Section V concludes the paper.

\section{RELATED STUDY}

In the following the related research activities on VANETs and other broadcast proposed techniques for the same are briefly described. The main topic of concern in this paper is the interference caused by the unnecessary messages received to a node during movement in a network. Each access point or node while moving on a road broadcasts some messages about its location or nearby activities to its neighboring nodes to avoid accidents or to give traffic related information. But it might be possible that not all the nodes receiving those messages might need them and so those messages may prove to be waste for them congesting their record and thus delaying the acknowledgement of useful or some important message. This becomes a matter of concern in case of an emergency. Thus it becomes important to reduce the exchange of as much number of unnecessary messages as we can. To accomplish this various studies have been done. [1] Few researchers suggest the MAC protocol in combination with the clustering algorithm. In the clustering algorithm suggested in [1] the mobile nodes form a cluster and the most stable node is made the cluster head. It becomes cluster head's responsibility to send a message to the node of another cluster via the cluster head of that cluster. The cluster head loses its energy with time and so a new cluster head is selected by evaluating the energy of each node in the cluster. In most of the studies clustering algorithm is taken as a base for reducing the interference in network or to maximize the stability and efficiency of the network. [2] Studies also specify that maximizing the life span of a cluster head can increase a network's stability and efficiency thus improving the complete structure. By increasing the time span for which a node a remains a cluster head the number of times a cluster head is selected in a particular duration is decreased and this also means that the system is stable for a longer duration. [3] It was also found that also by altering the message broadcasting techniques the network traffic can be reduced. It was suggested that by using $15 \mathrm{P}$ and slotted $15 \mathrm{P}$ algorithm we can reduce the number of messages passed. These algorithms tell that every node broadcasts a message to all its neighboring nodes and the new node that receives this message will also broadcast it further so by reducing the broadcasting range of any node the repetitive delivery of the same message to a common node can be avoided. It was also suggested that by doing probabilistic calculations and then sending messages according to those calculations can also reduce the message load on nodes. Therefore by analyzing and combining various studies we found that by changing the basic $\mathrm{CH}$ selection procedure we could reduce the messages and thus reduce the load. This could be helpful as a complete set of messages is being removed from the system which could be implemented otherwise as well and in turn also increases the time span of the cluster head increasing its stability. The idea with its advantages is explained in detail in the further sections.

\section{PROPOSED REDUCTION TECHNIQUE}

This section of the paper is going to talk about making the access points as the cluster heads. The access points are small devices fitted at the roadsides or on the dividers (middle of two opposite direction roads) at certain distance to provide information sharing. The vehicles share and access the traffic information through the access points. The use of access points improves the efficiency of the ad hoc network to share information in the network.

$\mathrm{CH}$ is the most stable node of the cluster formed. $\mathrm{CH}$ should have high speed internet connectivity so that there is no delay in message passing. So, the access points can be made the $\mathrm{CH}$. Now, each node gets connected to the access points as it enters into its range. The access points are in turn connected to the routers which keep certain information regarding the nodes that are within their range. A group of three to four access points are connected to one single router. Figure 2 depicts the same

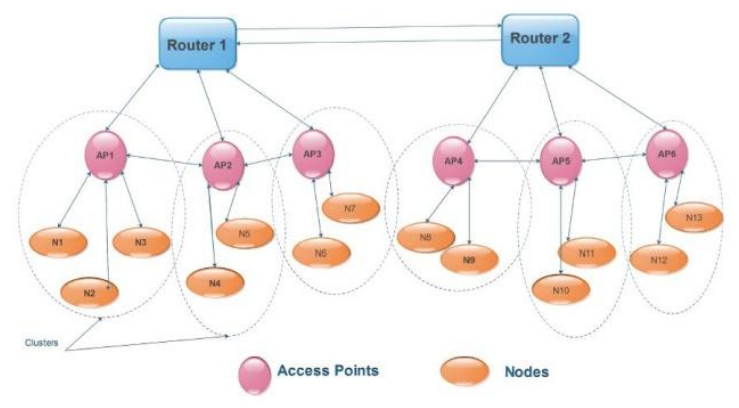

Fig 2: Cluster formation in VANET

The information stored at the routers should be any unique id, with the help of which we can uniquely identify the nodes. For this, we can use the IP address of the node to be stored in the router's information record. Other than this, the routers should keep the information about the direction in which that specific node is moving (either from left to right or from right to left).

All this can be achieved in a very simple manner. Any node when enters into a cluster gets connected with an access point. These access points are connected with a router which assigns an IP address to the node. The routers maintain a list of the previously assigned addresses, and checks before assigning a new address to any node whether the address is assigned earlier or not.

Collecting information about the nodes again and again at each router is a tedious work. So to overcome this we can use the information from the previous router (say R1) which has already registered the node and has its information. As soon as the node is leaving the range of a router in which it had registered itself and is entering into another router's range(say R2), the previous router(R1) would transfer the information of that node to the new router(R2). Now, this router can use this information directly without wasting time in assigning address and storing the direction of the node. 


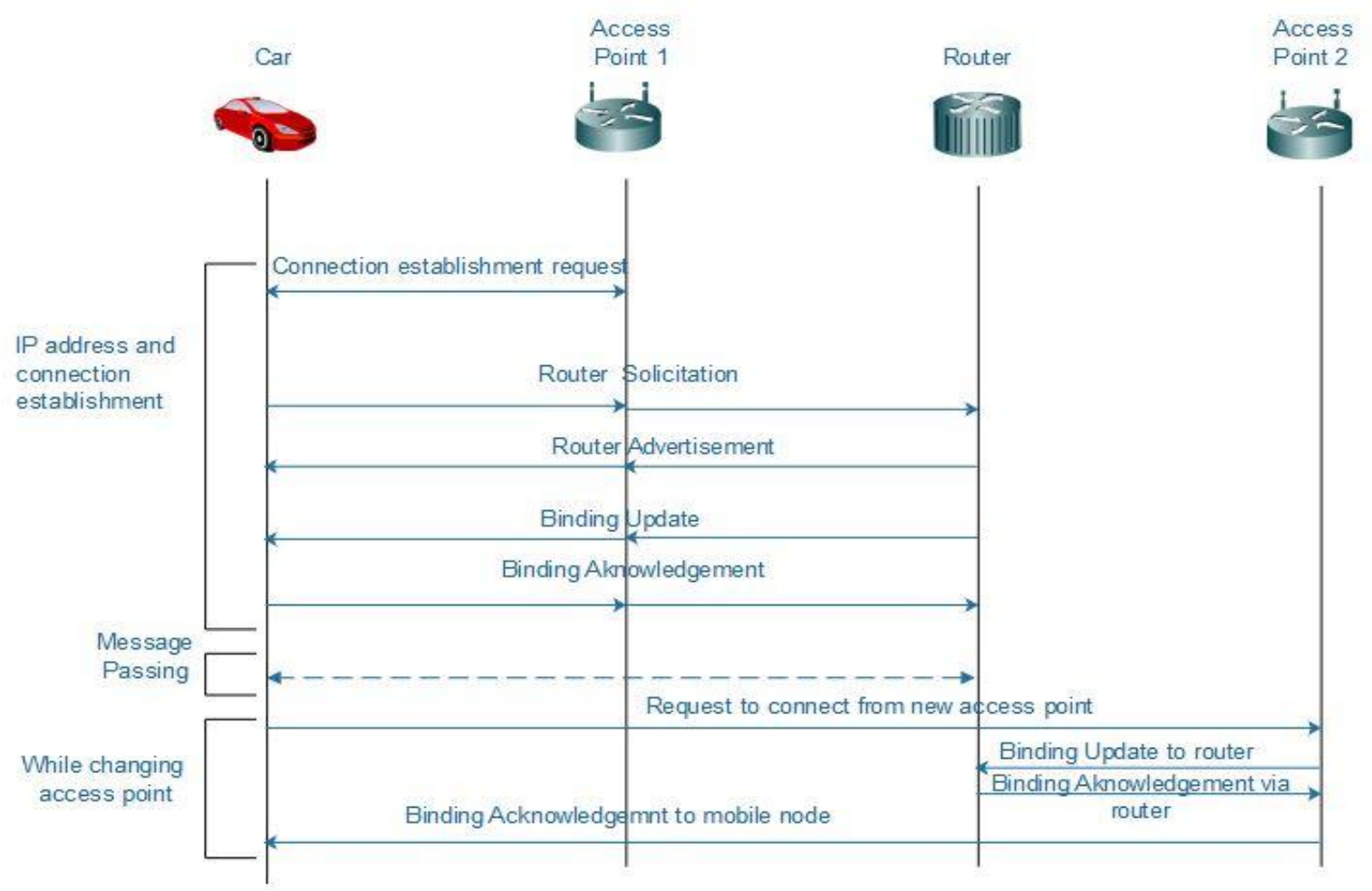

Fig 3: Communication within a single router

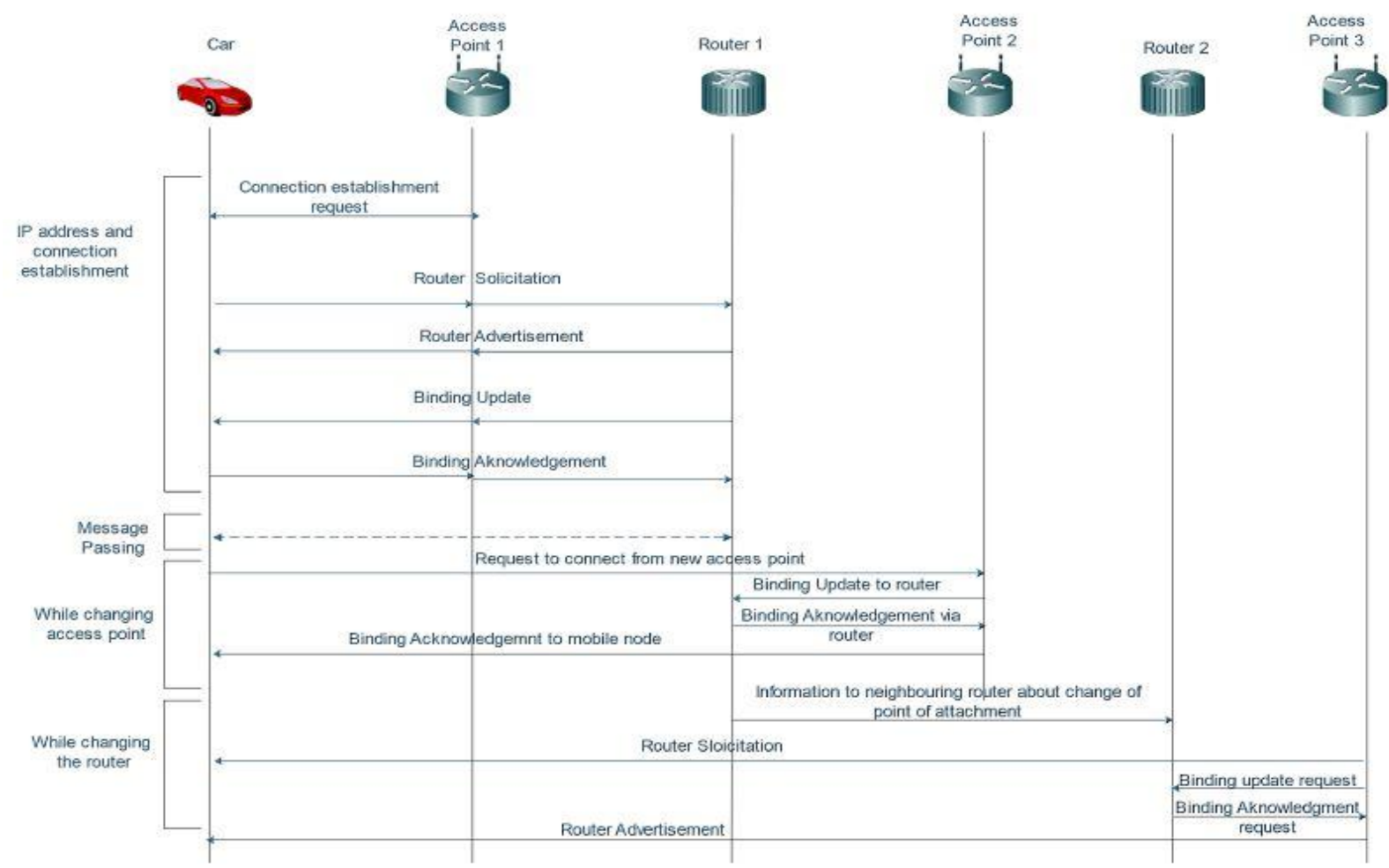

Fig 4: Inter router communication

\section{COMPARISON}

This technology is advantageous over the previous researches in the following prospects. It helps to reduce the interference in network without compromising with the security, speed, efficiency and message passing of the nodes. Various comparisons are given below.

\subsection{Stability}

The nodes in the cluster are able to conserve their energy as no more evaluation or calculation of their energy is required and thus a complete lot of message passing is avoided. This thus enhances the stability of the nodes. The $\mathrm{CH}$ is the most stable and highly connective node which is 
still conserved in this technique. The stability of the cluster thus enhanced improves the efficiency of the network.

\subsection{Packet loss}

Packet loss is an important matter of concern when it comes to mobile networks. Increased bandwidth and high speed Internet facility provided by the wired access points which are considered to be the CHs solves this problem. Since the access points are wired it gives us complete bandwidth availability and thus no risk of packet loss is left. Due to wired communication risk of packet loss is decreased, whereas in wireless communication the probability of the same is higher. Hence all the messages are safely transferred.

\subsection{Cluster Head Time Span}

Researchers have claimed that more the time span of $\mathrm{CH}$ more is the efficiency of the clustering algorithm which in turn increases the stability and performance. Since the access points are static thus making them as $\mathrm{CH}$ s increases the lifetime of the $\mathrm{CH}$ in a cluster as the overhead of choosing a new $\mathrm{CH}$ on the basis of changing stability of the nodes is eliminated. Thereby, decreasing the number of message exchanges in the network and thus decreasing interference.

\section{CONCLUSION}

Message passing is a very important issue when it comes to VANET. On time delivery of messages and decrease in the number of unnecessary messages is what is talked about in this paper. Earlier techniques had mobile $\mathrm{CH}$ due to which the energy of $\mathrm{CH}$ changed continuously that lead to the selection of new $\mathrm{CH}$ depending upon the energy of each node in the cluster. This complete model involved a lot of message exchanges which have been avoided in the proposed scheme. According to the proposed scheme stationary access points are made the $\mathrm{CH}$ and the routers that they are connected to assign and store the IP addresses assigned to each node in the cluster. Whenever a node moves from a cluster of one router to that of another the router depending upon the node's movement direction passes on the information of the node from its record table to the associated router which further passes it on to the access point $(\mathrm{CH})$.By making fixed $\mathrm{CH}$ messages passed reduced. This kind of approach will reduce the number of messages exchanged and the interference caused in VANET.

\section{REFERENCES}

[1] Reducing Interferences in VANETs by Dmitry Zelikman and Michael Segal, Senior Member, IEEE.

[2] A new stability based clustering algorithm (SBCA) for VANETs by Ahmed Ahizoune, Abdelhakim Hafid at Network Research Lab, University of Montreal, Canada.

[3] Broadcasting Message Reduction Methods in VANET by Davron Makhmadiyarov, Soojung HUr, Yongwan Park,Yeungnam University.

[4] Adaptive Message Passing in Vehicular Ad-hoc Networks by Vijay S Ukani, Jitendra Bhatia.

[5] Luciano Bononi and Marco Di Felice, "A Cross Layered MAC and Clustering Scheme for Efficient Broadcast in," in MASS'2007, 2007, pp. 1-8.

[6] Jason J. Haas and Yih-Chun $\mathrm{Hu}$, "Communication Requirements for Crash Avoidance," in VANET, Chicago, Illinois, USA, 2010.

[7] P. von Rickenbach, S. Schmid, R. Wattenhofer, and A. Zollinger, "A robust interference model for wireless ad-hoc networks," in Parallel and Distributed Processing Symposium, 2005, p. 8.

[8] Valery Naumov, Rainer Baumann, and Thomas Gross, "An Evaluation of InterVehicle Ad Hoc Networks Based on Realistic Vehicular Traces," in MobiHoc '06, 2006, pp. 108-119.

[9] Ray K. Lam and P. R. Kumar, "Dynamic Channel Reservation to Enhance Channel Access by Exploiting Structure of Vehicular Networks," in VTC, 2010.

[10] Ray K. Lam and P. R. Kumar, "Dynamic Channel Partition and Reservation for Structured Channel Access in Vehicular Networks," in VANET, Chicago, Illinois, USA, 2010.

[11] Jens Mittag, Florian Thomas, Jérôme Härri, and Hannes Hartenstein, "A Comparison of Single- and Multi-hop Beaconing in VANETs," in VANET, Beijing, China, 2009.

[12] Giovanni Resta, Paolo Santi, and Janos Simon, "Analysis of MultiHop Emergency Message Propagation in Vehicular Ad Hoc Networks," in MobiHoc, Monteeal, Quebec, Canada, 2007 